\title{
INTERVAL CALIBRATION MODEL OF MULTISENSOR SYSTEM
}

\author{
Alexander Voschinin ${ }^{1)}$, Nikita Skibitski ${ }^{2)}$ \\ 1) Prof, Doctor of Technical Sciences, FGUP “TSNIIATOMINFORM”, Russia, 127434, Moscow, Dmitrovskoye \\ Shosse, 2, P.O. Box 971, phone: 7(095) 777-96-29, fax: 7(095) 976-72-03, \\ e-mail: apv@ainf.ru \\ 2) Associate Professor, Ph.D., Moscow Power Engineering Institute (Technical University), Russia, 111250, Moscow, \\ Krasnokazarmennaya 14, phone: 7(095) 362-72-28, fax: 7(095) 362-89-38, \\ e-mail: SkibitskyNV@mpei.ru
}

\begin{abstract}
Problem of multisensor system calibration is of great importance in a number of applications. Most often the problem is solving by means of statistical methods using data of calibration controlled experiment. However, in many cases uncertainty and inaccuracy of experimental data more reasonably to express not in terms of random errors but in terms of known bounded absolute errors. For this case based on the introduced definition of "interval readings" interval calibration model is suggested. Within interval paradigm all calibration subproblems are reasonably solved including sensor sensitivity test, most accurate sensors subset selection and aggregate estimation of measurable variable uncertainty interval. There are given a numerical examples.
\end{abstract}

Keywords: - myltisensor system, calibration, interval model, fitting curve, inverse function, accuracy, selection

\section{PROBLEM DEFINITION}

Let be given an instrument assigned for measuring a single variable $\mathrm{x}$ by $\mathrm{m}$ sensors of different type with monotonic conversion functions $y j=f j(x), j=1 \ldots m$. As a rule, the calibration problem is solved on the base of calibration controlled experiment $\mathrm{F}=\{\mathrm{xi} ; \mathrm{yl} \mathrm{i} ; \ldots \mathrm{yji} \ldots \mathrm{ymi}, \mathrm{i}=1 \ldots \mathrm{n}\}$ with $\mathrm{n}$ trials.

Basically, decomposition of multisensor system calibration problem resulting in two subproblems: first, for each $j$-th sensor to determine the direct function $\mathrm{yj}=\mathrm{fj}(\mathrm{x})$ using experimental data and second, to define its inverse $\mathrm{x}=\mathrm{fj}-1(\mathrm{yj})$.

Mostly, these problems are solved in the frame of statistical approach by means of regression analysis $[1,2]$ supported by well-known and popular software. At the same time regression analysis is based on rather restricted assumptions concerning the type and the model of errors. Namely, it is usually supposed that controlled variable $\mathrm{x}$ is measured without errors and responses of sensors are additively mixed with normally distributed random errors. In practice, the additive model of errors is too simple and does not correspond to a number of real situations. Statistical approach to the calibration problem cannot involve into consideration non-statistical errors of observations including systematic errors of measurements, roundoff errors, a priory expert information, etc. Besides that there exists theoretical difficulty to inverse the random normally distributed value. For this reason statistical approach does not allow to estimate correctly the accuracy of calibration model under question.

To overcome above given demerits of statistical approach the aggregate interval calibration model is suggested below.

\section{GENERIC DEFINITIONS OF INTERVAL APPROACH}

An interval approach $[3 \div 8]$ introduced below is based on the definition of interval variable. Let be given some numerical constant or variable a whose exact value is unknown but there are given lower and upper bounds a-; $a+$ of its possible values. Then interval $[a]=[a-; a+]=\{a: a-\leq a \leq a+\}$ is called interval variable denoted as [a]. It is followed from given definition that true value of parameter a certainly belongs to the interval. Neither density function is specified on interval.

Depending on priory information related with uncertainty of parameter a different models can be applied for determining the interval [a]. Particularly, lower and upper bounds of unknown true value a can be derived from replications of trials and expert's estimation. Some times, there is given point observation $a$ with known either absolute error $\Delta$ or 
fractional error $\delta=\Delta / \mathrm{a}$. Then the lower and upper bounds of interval can be easily calculated. Thus, different forms of interval representation can be used

$$
[\mathrm{a}]=[a-\Delta ; a+\Delta]=[a \cdot(1-\delta) ; a \cdot(1+\delta)]=[\mathrm{a}-; \mathrm{a}+]
$$

Evidently, that there exists one-to-one correspondence between all representations of interval.

In present paper interval approach is applied to the calibration of multisensor system under assumption that errors of observations (both in $\mathrm{x}$ and y) are bounded and there are given $n$ observations of calibration experiment presented in the interval form $\mathrm{F}=\{[\mathrm{xi}] ;[\mathrm{y} 1 \mathrm{i}], \ldots[\mathrm{ymi}], \mathrm{i}=1 \ldots \mathrm{n}\}$.

\section{INTERVAL CALIBRATON MODEL FOR A SINGLE SENSOR}

Since multisensor calibration model is based on the calibration model of a single sensor let's consider it in more details.

Let be given $n$ interval observations of calibration experiment for a single sensor $\{[\mathrm{xi}]$; [yi], $\mathrm{i}=1 \ldots \mathrm{n}\}$ (see Fig. 1a). Using interval observations it is necessary to solve the following problems:

to test whether sensor is sensitive to the change of variable $\mathrm{x}$ or not;

if yes, to select the proper type of fitting curve;

to determine the parameters of direct and inverse interval calibration functions (models).

Testing of sensor sensitivity. Within the framework of interval approach any monotonic function $\mathrm{y}=\mathrm{f}(\mathrm{x})$ which passes through all intervals can be applied for fitting results of experiment. If there are number of such functions it is reasonably to select the simplest one. But if it is function like $\mathrm{y}=\mathrm{C}$, i.e. if there exists horizontal line that passes through all interval observations then sensor is not sensitive to the change of variable $\mathrm{x}$ and cannot be used for measuring. More strictly we can state the following

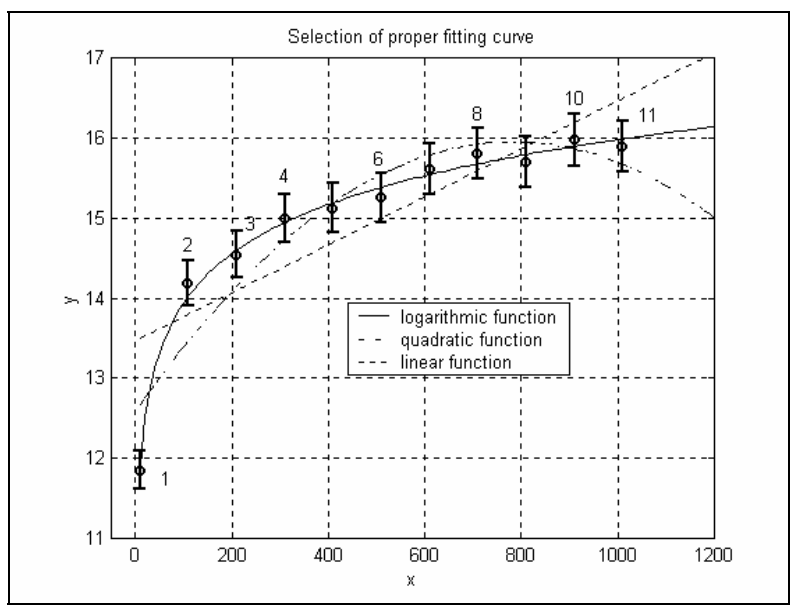

Fig 1 a.

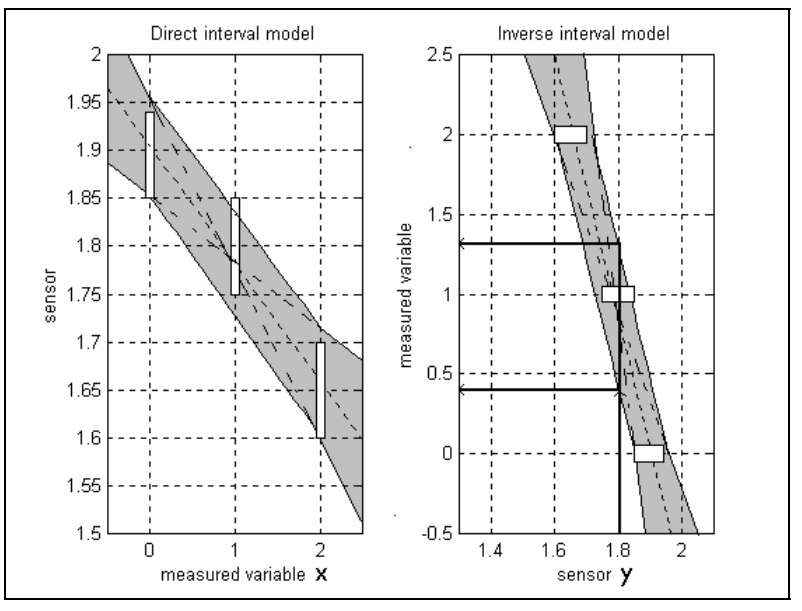

Fig $1 b$.

If there exists such constant $\mathrm{C}$ that $\mathrm{C} \in[\mathrm{yi}]$ for any i-th observation then sensor is not sensitive and should be cast out from the given set of sensors.

(With reference to fig.1 a such case may appear if calibration experiment contains only observation from No7 to No11).

Selecting of fitting curve type. Within suggested interval approach this problem can be easily solved when there are given some typical functionscandidates for fitting. For experimental data consisting of 11 interval observations presented in the fig. 1a three functions have been tested, namely, linear, quadratic and logarithmic. Their coefficients have been calculated with least square method (LSM) using midpoints of observation intervals. It is easy to see in Fig.1a that linear function intersects only 4 observations, quadratic function does not intersect observations No1, No2 and No3. Logarithmic function passes through all interval observations and for the experiment under consideration it is the only function from given set, which can be selected for fitting interval data.

Interval calibration model. To clarify interval approach to building calibration interval model we will use the data of simple real calibration experiment. In this experiment viscosity of samples $\mathrm{x}$ have been measured with spectrometer by readings of incident light $\mathrm{y}$. Viscosity was adjusted at three levels with normalized values $0,1,2$. Each level was replicated four times that produced the lower and upper bounds of each interval observation. Uncertainty in establishing the viscosity levels with absolute errors equal to 0.05 has been also adopted. Three resulting interval observations (shown in left subplot of Fig. 1b as white rectangles) are

$[\mathrm{x} 1]=[-0.05 ; \quad 0.05] ; \quad[\mathrm{y} 1]=\quad[1.85 ; \quad 1.94] ;$ $[\mathrm{x} 2]=[0.95 ; 1.05] ;[\mathrm{y} 2]=[1.75 ; 1.85] ;[\mathrm{x} 3]=[1.95 ;$ $2.05] ;[\mathrm{y} 3]=[1.6 ; 1.7]$.

First, to deliver the point estimation of measured variable $\mathrm{x}$ the average calibration model $\bar{x}$ is calculated with LSM using midpoints of given 
interval observations. Then, for specific extreme points of interval observations direct interval models are built as a linear interval spline-functions. These spline-functions describe bounds of variable y for three section of $\mathrm{x}$ total range, namely: No1: $0 \leq \mathrm{x} \leq 2$ (value of $\mathrm{x}$ within calibration experiment); No2: $\mathrm{x}<0$; No3: $\mathrm{x}>2$ (value of $\mathrm{x}$ out of calibration experiment). At the last stage inverse calibration models are calculated.

All mentioned models obtained for specific extreme points of given interval observations are presented in the Table 1.

At the second line of the Table 1 the direct yav and inverse $\bar{x}$ average models calculated for midpoints of interval observations $\{$ xiav, yiav $\}$ are presented. Direct models $y_{\text {in }}{ }^{-}(x)$ and $y_{\text {in }}{ }^{+}(x)$ for section No1 have been calculated with LSM for respective lower-left and upper-right corner points of interval observations. Boundary calibration models $x_{\text {in }}{ }^{-}(y)$ and $x_{\text {in }}{ }^{+}(y)$ have been defined by inverse of direct models.

Direct interval models $y_{\text {out }}{ }^{-}(x)$ and $y_{\text {out }}{ }^{+}(x)$ out of the range $0 \leq x \leq 2$ can be found similarly for the pair of observations with coordinates $\{(\mathrm{x} 1+; \mathrm{y} 1+)$; $(\mathrm{x} 3-; \mathrm{y} 3-)\}$ and $\{(\mathrm{x} 1-; \mathrm{y} 1-) ;(\mathrm{x} 3+; \mathrm{y} 3+)\}$. Inverse of these models produce respective models $x_{\text {out }}{ }^{-}(y)$, $x_{\text {out }}{ }^{+}(y)$ that give uncertainty intervals out of experimental range. (Respective extreme models are drawn in Fig.1b by dash lines). Resulting direct and inverse interval models displayed in Fig. $1 \mathrm{~b}$ by gray color reflect uncertainty interval of $y$ for any given $x$ and vice versa.

Table 1.

\begin{tabular}{|c|c|c|c|c|}
\hline \# & $\mathbf{R a}$ & $\begin{array}{l}\text { Specific } \\
\text { points }\end{array}$ & $\begin{array}{l}\text { Direct } \\
\text { model }\end{array}$ & $\begin{array}{c}\text { Inverse } \\
\text { model }\end{array}$ \\
\hline & & $\begin{array}{l}x_{i}^{a v}, y_{i}^{a v}, \\
\mathrm{I}=1,2,3 .\end{array}$ & $\begin{array}{l}y^{a v}=1.904- \\
0.1225 \cdot \mathrm{x}\end{array}$ & $\begin{array}{l}\bar{x}=15.544- \\
8.163 \cdot y\end{array}$ \\
\hline \multirow[t]{2}{*}{1} & \multirow{2}{*}{$\begin{array}{l}0 \leq x \leq 2 \\
1.6 \leq y \leq \\
1.94\end{array}$} & $\begin{array}{l}x_{i}^{-}, y_{i}^{-}, \mathrm{i}=1, \\
2,3 .\end{array}$ & $\begin{array}{l}y_{\text {in }}=1.852- \\
0.125 \cdot \mathrm{x}\end{array}$ & $\begin{array}{l}x_{\text {in }}{ }^{-}=14.816- \\
8 \cdot y\end{array}$ \\
\hline & & $\begin{array}{l}x_{i}^{+}, y_{i}^{+}, \mathrm{i}=1, \\
2,3 .\end{array}$ & $\begin{array}{l}y_{\text {in }}{ }^{+}=1.956- \\
0.12 \cdot \mathrm{x}\end{array}$ & $\begin{array}{l}x_{i n}+=16.3- \\
8.333 \cdot y\end{array}$ \\
\hline \multirow[t]{2}{*}{2} & \multirow[t]{2}{*}{$\begin{array}{l}x<0 \\
y>1.94 .\end{array}$} & $\begin{array}{l}x_{1}^{+}, y_{1}^{+} ; x_{3}^{-}, \\
y_{3}^{-} .\end{array}$ & $\begin{array}{l}y_{\text {out }}{ }^{+}=1.846- \\
0.0714 \cdot \mathrm{x}\end{array}$ & $\begin{array}{l}x_{\text {out }}=10.89- \\
5.588 \cdot y\end{array}$ \\
\hline & & $\begin{array}{l}x_{1^{-}}^{-}, y_{1}^{-} ; x_{3}^{+}, \\
y_{3}^{+}\end{array}$ & $\begin{array}{l}y_{\text {out }}-=1.949- \\
0.179 \cdot \mathrm{x}\end{array}$ & $\begin{array}{l}x_{\text {out }}{ }^{+}=25.85- \\
14 \cdot y\end{array}$ \\
\hline \multirow[t]{2}{*}{3} & \multirow[t]{2}{*}{$\begin{array}{l}x>2 \\
y<1.6 .\end{array}$} & $\begin{array}{ll}x_{l^{-}}^{-} & y_{1}^{-} ; x_{3}^{+}, \\
y_{3}{ }^{+}\end{array}$ & $\begin{array}{l}y_{\text {out }}=1.949- \\
0.179 \cdot \mathrm{x}\end{array}$ & $\begin{array}{l}x_{\text {out }}{ }^{-}=25.85- \\
14 \cdot y\end{array}$ \\
\hline & & $\begin{array}{l}x_{1}^{+}, y_{1}^{+} ; x_{3}^{-}, \\
y_{3}^{-}\end{array}$ & $\begin{array}{l}y_{\text {out }}{ }^{+}=1.846- \\
0.0714 \cdot \mathrm{x}\end{array}$ & $\begin{array}{l}x_{\text {out }}{ }^{+}=10.89- \\
5.588 \cdot y\end{array}$ \\
\hline
\end{tabular}

Average calibration model $\bar{x}$ and interval calibration model $x^{-}(y), x^{+}(y)$ allow to determine both point estimation $\bar{x}$ and interval of uncertainty of $\mathrm{x}$ for any given value $\mathrm{y}$. For example, for $\mathrm{y}=2$ we get $\bar{x}=-0.78,[\mathrm{x}]=[-2.153 ;-0.28]$. For $\mathrm{y}=1.8$ we have $\bar{x}=0.85,[\mathrm{x}]=[0.42 ; 1.3]$. (Graphically, uncertainty interval for the last case is shown in the right subplot of fig. 1b).

It is necessary to stress that value $\bar{x}$ may not coincide with midpoint of uncertainty interval and the fact that out of the experimental range of $x$ the width of uncertainty interval may increase very fast.

\section{AGGREGATED INTERVAL CALIBRATION MODEL}

Suppose now that there is given set of $\mathrm{m}$ sensors, interval data of calibration experiment $\mathrm{F}=\{\mathrm{xi}$; [y1i],...[ymi], $i=1 \ldots n\}$ and all sensible to the measuring value $\mathrm{x}$. To build the calibration model of multisensors system it is necessary to solve the following problems:

to estimate the parameters of all direct functions $y j=f j(x), j=1 \ldots m$.

to determine all inverse functions $\mathrm{x}=\mathrm{fj}-1(\mathrm{yj})$, $\mathrm{j}=1 \ldots \mathrm{m}$.

to select the subset of the most accurate sensors;

to build aggregated interval calibration model for chosen sensors.

Since solution of two first problems is described above let's consider the two last problems supposing that all direct and inverse models are already obtained.

Selection of the most accurate sensors subset can be fulfilled with following simple algorithm:

For given experimental values of $x i(i=1 \ldots n)$ and known direct average models fjav(xi) point estimates $\quad \mathrm{jjiav}=\mathrm{fjav}(\mathrm{xi}) \quad(\mathrm{i}=1 \ldots \mathrm{n}, \mathrm{j}=1 \ldots \mathrm{m})$ are calculated;

Using points estimates yjiav and inverse models $x_{i n}{ }^{-}\left(y_{j i}^{a v}\right), \quad x_{i n}{ }^{+}\left(y_{j i}^{a v}\right)$ uncertainty intervals [xi]j $(\mathrm{i}=1 \ldots \mathrm{n}, \mathrm{j}=1 \ldots \mathrm{m})$ are calculated for each sensor.

Selection of the most accurate sensors subset is based on the following statements:

Sensor $\mathrm{No}(\mathrm{w})$ is the worst one and have to be eliminated from the set of $\mathrm{m}$ sensors if intervals $[x i] w$ covers all the rest intervals $[x i] j, j=1 \ldots m, j \neq w$ for any fixed i-th observation.

Sensor No(b) is the best one if interval [xi]b for any fixed $i$-th observation is enclosed in all the rest intervals $[x i] j, j=1 \ldots m, j \neq b$. In this case all the rest sensors can be excluded from the given set and multisensors system degenerates into system with a single sensor $\mathrm{No}(\mathrm{b})$.

If within the set of sensors there is neither worse nor best one all sensors are equivalent and can be used for building aggregate interval calibration model.

Aggregate estimate of uncertainty interval $[\mathrm{x}]$ is 
formed using intervals $[\mathrm{x}] \mathrm{j}$ for all $\mathrm{m}$ sensors obtained with inverse model for some fixed unknown value $\mathrm{x}$ common for all sensors. Three aggregated estimates can be used: intersection of intervals $[x]_{\cap}$; mean of intervals $[\bar{x}]$ and union of intervals $[x]_{\cup}$ which are defined as:

$$
\begin{gathered}
{[x]_{\cap}=\left[\max _{j}\left(x_{j}^{-}\right) ;\left[\min _{j}\left(x_{j}^{+}\right)\right] ;\right.} \\
{[\bar{x}]=\frac{1}{m} \sum_{j=1}^{m}\left[x_{j}\right] ;[x]_{\cup}=\left[\min _{j}\left(x_{j}^{+}\right) ; \max _{j}\left(x_{j}^{-}\right)\right] .}
\end{gathered}
$$

Aggregate estimates (2) give the different degree of certainty. Estimate $[x]_{\cup}$ produces the widest uncertainty interval in turn estimate $[x]_{\cap}$ gives the most narrow uncertainty interval. It possible to show that estimate $[x]_{\cap}$ tends to the true value $\mathrm{x}$ if there are no systematic errors and number of observation replicates in calibration experiment tends to infinity.

To illustrate selecting technique we will use the data of real calibration experiment [1]. In this experiment five sensors have been used for measuring level of viscosity with four different spectrometers (their responses are denoted as y1, y2, $\mathrm{y} 3, \mathrm{y} 4)$ and one goniofhotometer recording peakheight (response y5). At the calibration experiment viscosity have been adjusted at three levels - 0, 1, 2 (normalized values). Each level was replicated four times.

Using interval data of calibration experiment direct and inverse interval models have been calculated for all five sensors in accordance with methodology described above. Then using midpoints yjiav of sensor's readings for each of three viscosity levels uncertainty intervals [xij] of viscosity have been calculated. Results are shown in the fig. 2 .

In fig. 2 sensors are numbered and their boundary readings marked with different marks jointed together by vertical lines. Each of these line segments is interval of uncertainty [xji] produced by errors of respective sensor.

Be guided by the above statements it is easy to see that sensor No5 is the worst, all the rest are equivalent. The estimates (2) for subset of four chosen sensors when $\mathrm{x}=1$ are equal respectively to

$$
\begin{aligned}
{[x]_{\cap} } & =[0.82 ; 1.01] ; \quad[\bar{x}]=[0.64 ; \quad 1.27], \\
{[x]_{\cup} } & =[0.44 ; 1.75] .
\end{aligned}
$$

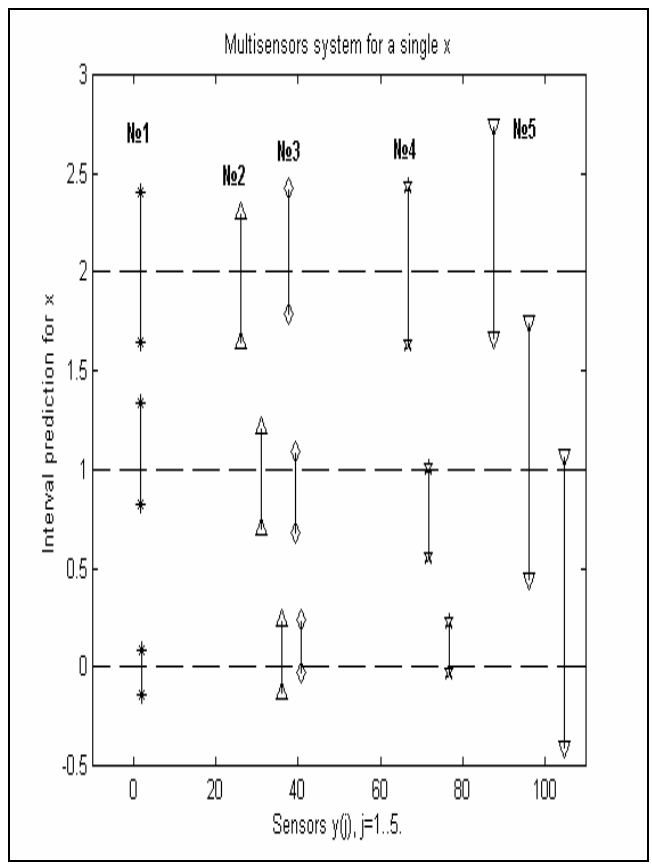

Fig.2

\section{CONCLUSION}

Application of statistical methods to the calibration problem is faced with a number of difficulties that followed from the restricted initial assumptions concerning model of errors. Statistical approach does not allow to involve correctly into consideration the errors of non-statistical type and a priory expert information. Interval approach which has no such restriction can be seen as an alternative to the statistical methods. Its application to the calibration of multisensors system allows not only to take into account information of any type but also to deliver meaningful and reliable results based on simple methodology and algorithms.

\section{REFERENCES}

[1] P.J. Brown, Multivariate calibration, J. R. Statist.Soc.B, 3. 1982. p. 44. N3.

[2] L. Smith, M. Corbett. Measuring Marathon courses: An application of statistical calibration theory. - Appl.Statist., 1987, 36, N3

[3] R.Moore. Interval Analysis, Englewood, Cliffs, N.Y., Prentice-Hall, 1966.

[4] A. Voshchinin, S. Simoff, N. Divak. Design of Experiments and Data Analysis: New Trends and Results, ANTAL, Moscow, 1993, p. 11-51

[5] A. Voschinin, G. Sotirov. Optimization Under Uncertainty, Moscow Sofia, 1989, 320 pp. (in Russian).

[6] A. Voschinin. Interval Approach to the Problems of Hypothesis Testing and Estimation of parameters implicit functions, Moscow, Industrial Laboratory, vol. 7, 2000 (in Russian).

[7] V. Gorsky, T. Shvetzova-Shilovskaya, A. 
Voschinin. Risk assessment of accident involving environmental high-toxicity substances, Journal of Hazardous Materials, No78, 2000.

[8] A. Voshchinin. Interval Analysis: Development and Perspectives, Moscow, Industrial Laboratory, vol. 1, 2002 (in Russian)

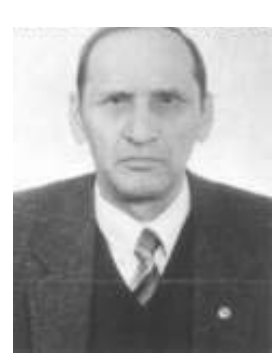

Alexander Voshchinin. Graduated from the Moscow Power Engineering Institute Technical University (MPEl), Department of Control Systems. 1965-1993 - Assistant Professor, Associated Professor, Professor, Dr. -Sc. (Control Systems), MPEl, Department of Control Systems.

1994-1997 - Professor of Applied Mathematics and Statistics, the Meadle East Technical

University, Ankara - Gaziantep, Turkey.

At present - Chief Scientist, Central Research Institute of Management, Economics \& Information,

Ministry of Atomic Energy of Russia (MINATOM), Moscow.
Member of Russian Nuclear Society;

Member of Editorial Board of the Journal Industrial Laboratory;

Expert of MINATOM on Methodologies \& Tools for Energy Analysis.

Areas of interests: Methodologies \& Tools for Energy Analysis, Least-Cost Planning of Energy

Systems, Financial Risk Analysis, Decision-making under uncertainty, Interval Models of Uncertainty.

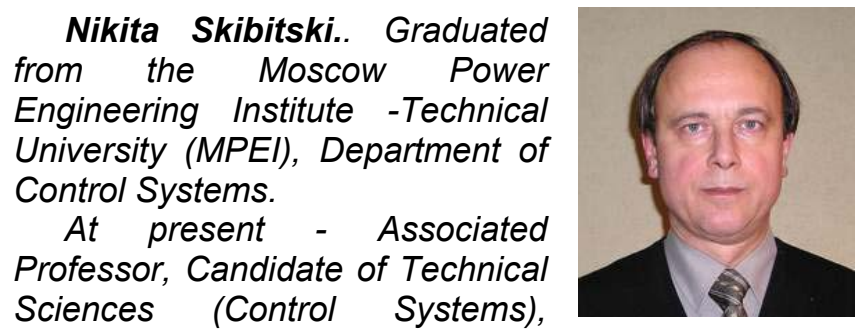

Department of Control Systems, MPEI, Head of the Department on Commerce and Trade, MPEI.

Areas of interests: Methodologies \& Tools for Technical System Analysis, Statistics, Calibration models, Interval Models of Uncertainty. 